INTERNATIONAL JOURNAL OF RESEARCHES IN BIOSCIENCES, AGRICULTURE AND TECHNOLOGY

(C) VISHWASHANTI MULTIPURPOSE SOCIETY (Global Peace Multipurpose Society) R. No. MH-659/13(N) www.vmsindia.org

\title{
ELECTRICAL CONDUCTIVITY OF ELECTRODEPOSITED THIN FILM OF POLYPYRROLE/CNT NANOCOMPOSITES
}

\author{
P. D. Burghate1, R. Mahore ${ }^{2}$, D. K. Burghate ${ }^{2}$ and S. Kondawar ${ }^{3}$ \\ ${ }^{1}$ Department of Chemistry, Shri Shivaji Science College, Nagpur \\ ${ }^{2}$ Department of Physics, Shri Shivaji Science College, Nagpur \\ ${ }^{3}$ Department of Physics, R.T.M. Nagpur University, Nagpur \\ pratibhaburghate@rediffmail.com
}

\begin{abstract}
:
The conducting polypyrrole / functionalized carbon nanotubes (PPy/CNT) nanocomposites have been electrochemically deposited on stainless steel using $0.1 \mathrm{M}$ aqueous solution of dodecylbenzene sulfonic acid (DBSA). 10 wieght percentage of functionalized MWCNTs with respect to pyrrole was used to fabricate nanocomposites. The structure and morphology of the nanocomposites were studied by UV-Vis spectroscopy, Fourier transform infrared spectroscopy (FT-IR), and scanning electron microscopy (SEM).The composites showed good interaction based on the shift to shorter wavelengths in the electronic transition, indicating the interaction between PPy and functionalized CNTs. The SEM images revealed the porous rough and granular morphology of pure polypyrrole whereas tubular form of nanocomposites indicating polypyrrole coating on CNTs. The electrical conductivity increases as the CNT \% increases thus indicating that composites enhanced properties as compared to pure polypyrrole.
\end{abstract}

Keywords: Conducting polypyrrole, Carbon nanotubes (MWCNT), Electrodeposition, Nanocomposites

\section{Introduction:}

Nanocomposite of carbon nanotubes with material such as conducting polymer are very attractive combinations for the development of electrochemical sensors, biosensors gas sensorsand energy storage devices. Carbon nanotubes (CNTs) exhibit excellent mechanical, electrical, and magnetic properties as well as nanometer scale diameter and high aspect ratio, which make them an ideal reinforcing agent for high strength polymer composites [1]. Several studies in the fields of new materials have introduced the possibility to use Conducting Polymers as suitable matrices to disperse nanostructural elements, such as carbon nanotubes (CNTs). It has been shown that the introduction of CNTs into a polymer matrix can improve the electrical conductivity and the mechanical properties of the original polymer matrix [2]. Among various conducting polymers, polypyrrole (Ppy) as an intelligent material plays an important role in the electrochemical sensors, biosensorsgas sensors and energy storage devices. The versatility of this polymer is determined by its capability to protect electrodes from interfering material, and easy way for electrochemical deposition on the surface of any type of electrode. Ppy films can be easily formed from aqueous solutions by electrochemical routes at neutral $\mathrm{pH}[3]$.

In the present work pure conducting polypyrrole and composite consisting of conducting polypyyrole, carbon based material (CNT) was synthesized by electrodeposition method. By making the above said pure and composite it is intended to exploit the advantages of individual material. The conducting polymers can offer better interparticle connectivity to the CNT in the composite could obviously offer high electronic conductivity and could also facilitate the easy access of the electrolyte into the bulk film by means of its mesopore. [4] Hence, such a composite approach is expected to offer much improved electrochemical performance of the material, which is often difficult to achieve from either the conducting polymer or CNT

\section{Material and Methods:}

\subsection{Chemical \& Reagents}

Pyrrole (with 98\% purity) from Sigma-Aldrich, Multiwalled Carbon nanotubes (CNT) from NCL Laboratory Delhi, with size $2-6 \mathrm{~nm}$ and length $0.1-10 \mu \mathrm{m}$ with $90 \%$ purity ; dodecyl benzenesulphonic acid (sodium salt) fromMerck. All the other reagents were of analytical grade. All solutions were prepared using triple distilled water.

\subsection{Characterization}

The morphology of the nanocompositewas studied using scanning electron microscopy (SEM). The infrared spectrum of the nanocompositewas obtained using Bruker Alpha FT-IR spectrometer. The absorption spectra of the nanocomposite obtained from a UV-Vis spectrophotometer Shimadzu-UV1800 .

\subsection{Experimental}

Electrochemical synthesis of Polypyrrole $/ f$ MWCNT nanocomposite film were performed in a one-compartment three-electrode glass cell at room temperature $\left(27{ }^{\circ} \mathrm{C}\right)$ using a Electrochemical analayser Model CH-600D. The 
stainless steel plate was used as a working electrode, platinum wire as a counter electrode and $\mathrm{Ag} / \mathrm{AgCl}$ was used as a reference electrode. To perform the experiment MWCNT were functionalized with carboxylic acid groups by sonication in a 3:1 sulfuric-acid/nitric-acid mixture for $3 \mathrm{hrs}$. Subsequently, the pretreated CNT were washed with water, then with $0.1 \mathrm{M}$ $\mathrm{NaOH}$ (to reach neutrality of $\mathrm{pH} 7.0$ ), filtered, and dried overnightat $80^{\circ} \mathrm{C}$. The functionalized MWCNT (10\% by weight of pyrrole) were dispersed in 0.1 M DBSA aqueous solution and sonicated for $1 \mathrm{~h}$. Pyrrole $0.1 \mathrm{M}$ was dissolved in this solution under ultrasonic stirring for 15 $\min$ at room temperature. Then, a nanocomposite films were electrochemically synthesized by electrolyzing this medium on a steel electrode. This grown Ppy/ $f$-MWCNT films was washed repeatedly with distilled water and methanol to remove the electrolyte and the monomer and then dried at room temperature under vacuum. Pure pyrrole was also polymerized by electrolysis of the aqueous solution of $0.1 \mathrm{M}$ DBSA and $0.1 \mathrm{M}$ pyrrole.

\section{Result and Discussion:}

Scanning electron microscopy (SEM) was used for examining the morphology of the Ppy $/ f$ MWCNT film. As one can see, from the images of Ppy/ $f$-MWCNT composites in Fig 1(b), the composites exhibit well dispersed carbon nanotubes enwrapped uniformly with Ppy. This suggests that the interaction between polymer molecules and MWCNTs overcomes the Van der Waals interaction between MWCNTs, which generally otherwise would result in separate growth or aggregates of Ppy. Such morphology is in agreement with early studies of conductingpolymer/CNT composites [5]. As a result, MWCNTs are more uniformly dispersed in the composite film prepared by mixing MWCNT with aqueous DBSA solution

FTIR spectra showed the main characteristic peaks at $675 \mathrm{~cm}^{-1}$ corresponding to ring deformation, $929 \mathrm{~cm}^{-1}$ corresponding to $\mathrm{C}-\mathrm{H}$ deformation, $1043 \mathrm{~cm}-1 \quad \mathrm{C}-\mathrm{H}$ in-plane bend, $1188 \mathrm{~cm}-1$ ring breathing, $1383 \mathrm{~cm}-1$ and $1350 \mathrm{~cm}^{-1}$ corresponding to the fundamental vibrations of polypyrrole ring, The peak at 1585

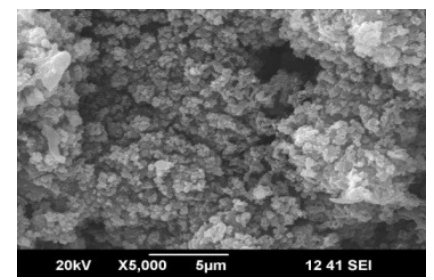

$\mathrm{cm}^{-1}$ corresponding to $\mathrm{C}=\mathrm{C}$. The peak at 3348 $\mathrm{cm}^{-1}$ corresponds to the $\mathrm{N}-\mathrm{H}$ bond. This agrees well with the ones available the literature, confirming the formation of Polypyrrole. The above mentioned peaks in the FTIR spectra of PPY/CNT showed the other important IR bands observed in Fig 1(b) are at $2813 \mathrm{~cm}^{-1}$ and 2656 $\mathrm{cm}^{-1}$ which are due to the $\mathrm{C} \mathrm{H}$ stretching vibrations and strong peak at $761 \mathrm{~cm}^{-1}$ is due to the $\mathrm{C} \mathrm{H}$ bending, which are the nanotube backbone vibrations thus confirming the formation of polypyrrole composite with CNT. $[6,7]$

The absorption spectra of the samples obtained from a UV-Vis spectrophotometer (Shimadzu-UV1800) are shown in Fig 3. The measurements of absorption spectra were carried out at room temperature for visible wavelength ranging from $400 \mathrm{~nm}$ to $800 \mathrm{~nm}$. The spectrum of polypyrrole shows two distinct bands at $294 \mathrm{~nm}$ and $420 \mathrm{~nm}$. These two transitions corresponded to the transitions from valence bond to polarons and bipolarons of the oxidized form of polypyrrole. The characteristics band for the polaronic transition around 420nm and the peak around $294 \mathrm{~nm}$ represents the $\Pi$ $\Pi^{*}$ electron orbital transition along the backbone of the polymer chain.As CNT's were incorporated with polymer absorption spectra as shown in Fig 3 the characteristic peak assigned to the polaron- $\Pi^{*}$ transition of polymer chain shifted to shorter wavelengths, indicating the interaction between polymer chain and CNTs. The $\Pi-\Pi^{*}$ transition of polymer in the nanocomposites was also shifted to shorter wavelengths. [8]

The electrical conductivity of the films studied at room temperature on thin film conductivity meter. By using the following formula

Where $\rho 0=\frac{v}{I} 2 \pi s$

$$
\rho=\frac{\rho 0}{G 7 \frac{w}{s}}
$$

$\mathrm{S}=$ thickness of sample

Distance between the pole i.e. $2 \mathrm{~mm}$

The conductivity of pure pyrrole was found to $0.5 \mathrm{~S} / \mathrm{cm}$ and the conductivtyofpolypyrrolecnt composite was found to be $1.3 \mathrm{~S} / \mathrm{cm}$.

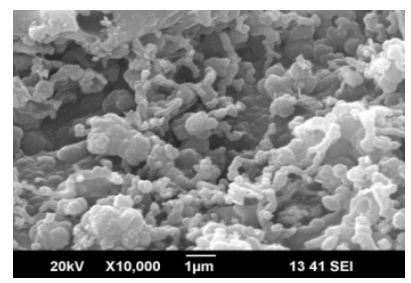

Figure. 1-SEM images of Ppy (a), Ppy/ $f$-MWCNT (b). 

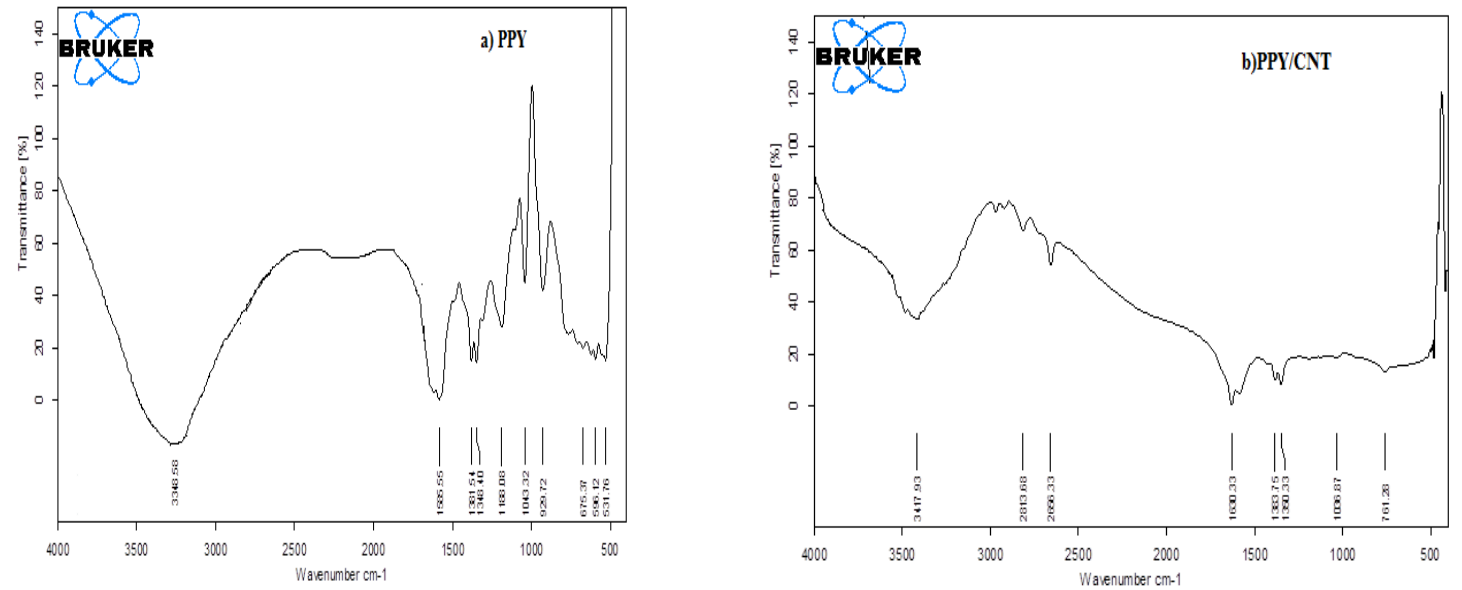

Figure. 2- FTIR spectra of (a) Pure Polypyrrole (PPY) (b) PPY/CNT

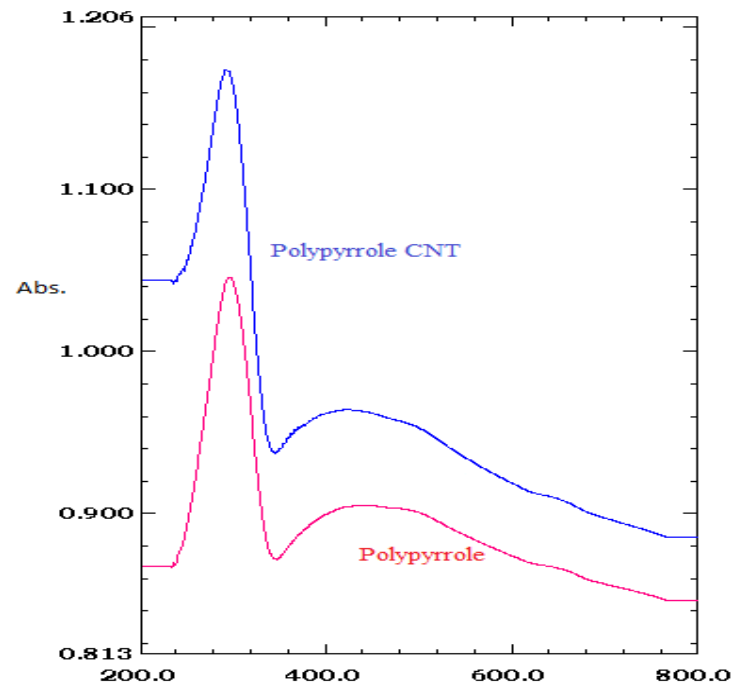

Figure. 3-Absorbance spectrum of the Ppy/MWNT for different MWNT

\section{Conclusion:}

Pure polypyrrole and Polypyrrole /CNT were successfully synthesized and the structural study of film was carried out by SEM, FTIR, UVVIS. The SEM results reveals the rough, porous and granular morphology. The composites showed good interaction based on the shift to shorter wavelengths in the electronic transition,

indicating the interaction between PPY and functionalized CNTs as observed in their UV-VIS and FTIR spectra. The composites with CNT shows enhanced conductivity as compared to pure polypyrrole.

\section{Acknowledgement:}

The authors gratefully acknowledge UGC,New Delhi(India) for the financial assistance to carry out this work through major Research Project file no. 41-931/2012(SR).

\section{References:}

[1] R.K. Sharma, B. Tiwari, J. S. Tomar,Advanced Engineering and Applied Sciences: An International Journal, 2013; 3(3): 28-30

[2] A Imani, G. Farzi and A.Ltaief ,International Nano Letters2013, 2013, 3:52

[3] M. A. Chougulea, S. G. Pawara, P. R. Godsea, R. N. Mulika, S.Senb, V. B. Patila, Soft Nanoscience Letters, 2011, 1, 6-10

[4] R.Mahore, D.K. Burghate, S.B.Kondawar,Proceedings, Advance Material World Congress-2013, Turkey

[5] S. Paul, Y. S. Lee, J. A Choi, Y. C. Kang, and D. W.Kim,Bull. Korean Chem. Soc. 2010,Vol. 31,No.

[6] S. Paul, K.S.Choi, D. J. Lee, P. Sudhagar, Y.S. Kang ,78 (2012) 649- 655

[7] R. Turcu, Al. Darabont, A. Nan, N. Aldea, D. Macovei, D. Bica, L. Vekas, O. Pana, M. L. 
Soran, A. A. Koos, L. P. Biro, Journal of Optoelectronics And Advanced Materials Vol. 8, No. 2, April $2006643-647$
[8] T. Ming Wu, H. L. Chang, Y. W. Lin, Composites Science and Technology 69 (2009) 639-644 